\title{
Equivalence of Deterministic walks on regular lattices on the plane
}

\author{
Ana Rechtman \\ IRMA, Université de Strasbourg, \\ 7 rue René Descartes, 67084 Strasbourg, France* \\ Raúl Rechtman \\ Instituto de Energías Renovables, \\ Universidad Nacional Autónoma de México, \\ Apdo. Postal 34, 62580 Temixco Mor., México ${ }^{\dagger}$
}

\begin{abstract}
We consider deterministic walks on square, triangular and hexagonal two dimensional lattices. In each case, there is a scatterer at every site that can be in one of two states that force the walker to turn either to his/her immediate right or left. After the walker is scattered, the scatterer changes state. A lattice with an arrangement of scatterers is an environment. We show that there are only two environments for which the scattering rules are injective, mirrors or rotators, on the three lattices. On hexagonal lattices, B. Z. Webb and E. G. D. Cohen [1], proved that given an initial position and velocity of the walker and an environment of one type of scatterers, mirrrors or rotators, there is an environment of the other type such that the walks on both environments are equivalent, meaning they visit the same sites at the same time steps. We prove the equivalence of walks on square and triangular lattices and include a proof of the equivalence of walks on hexagonal lattices. The proofs are based both on the geometry of the lattice and the structure of the scattering rule.
\end{abstract}

\section{INTRODUCTION}

In 1912 Paul and Tatiana Ehrenfest published a monograph on the foundations of statistical mechanics in the Encyklopädie der Mathematische Wissenschaften where among other subjects they presented the wind-tree model to explain Boltzmann's transport equation [2]. The wind is formed by particles that do not interact between themselves and move with the same speed along the $x$ and $y$ axes. The trees are fixed squares randomly placed on the plane with their diagonals aligned along the $x$ and $y$ axes that scatter the wind particles an angle of $\pm \pi / 2$. The motion of the wind particles is deterministic and time reversible. The model is a Lorentz gas so one can consider only one particle. The wind-tree model served as a starting point for the study of Lorentz lattice gases where a particle advances from a site to one of its nearest neighbor sites on a lattice in one time step and the trees, or better scatterers, occupy one site. Following Bunimovich we speak of walks on an environment of scatterers [3]. On a square lattice $\mathbb{Z}^{2}$ the trees became two types of mirrors, small line segments at angles of $\pi / 4$, a right mirror, and $3 \pi / 4$, a left mirror, with respect to the positive $x$ axis. In the flipping model, the scatterers flip from one orientation to the other one, after the particle is scattered $[4,5]$. Right (left) rotators have also been studied where the walker is scattered an angle of $\pi / 2$ to his/her right (left) on a square lattice [6-8]. Walks on two dimensional triangular lattices $\mathbb{T}^{2}[3,9-12]$ and on hexagonal lattices $\mathbb{H}^{2}[1,13,14]$ have been studied extensively.

We consider deterministic discrete walks on two dimensional regular lattices where the walker moves with unit

\footnotetext{
* rechtman@math.unistra.fr

† rrs@ier.unam.mx
}

speed from a site to a nearest neighbor site in one time step. All the sites are occupied by a scatterer that flips from right to left and vice versa after the walker is scattered. A walker on a flipping mirror environment in $\mathbb{Z}^{2}$ at a site $(x, y)$ is reflected by a mirror an angle of $\pm \pi / 2$ and jumps with unit speed to one of two nearest neighbor sites of $(x, y)$. The mirror at $(x, y)$ changes orientation as the walker passes by rotating an angle of $\pi$. In a rotator environment in $\mathbb{Z}^{2}$, the walker at $(x, y)$ turns an angle of $\pi / 2$ to his/her right (left) when there is a right (left) rotator at $(x, y)$ and jumps to the nearest neighbor site in front of him/her. The rotator at $(x, y)$ changes from right to left or left to right as the walker passes. The change in orientation of the mirrors or rotators, referred to as a flip, forbids the existence of closed orbits [12].

A walker on an environment initially filled with right mirrors in $\mathbb{Z}^{2}$ moves in a zigzag, alternating between a vertical step and a horizontal one with a speed of $\sqrt{2} / 2$, as we show in Fig. 1 (a). The initial velocity of the walker determines on which of the four diagonals he/she will move. On the other hand, a walker on an environment initially filled with right rotators in $\mathbb{Z}^{2}$ moves around his/her starting point and after 9,977 time steps, advances two sites horizontally and two vertically every 104 time steps in what is known as a highway with a speed of $\sqrt{2} / 52$ as we show in Fig. 1 (b) $[7,8,15,16]$. The initial velocity of the walker determines the direction of the highway.

Walks on both environments are also different on initially disordered environments. What is maybe more striking is that if the environment is disordered in some region and ordered in another one as in Fig. 2, the walker in the mirror environment will eventually leave the disordered region and move alternatively one site horizontally and the next vertically, Fig. 2 (a). The walker in the rotator environment will eventually leave the disordered 
(a)

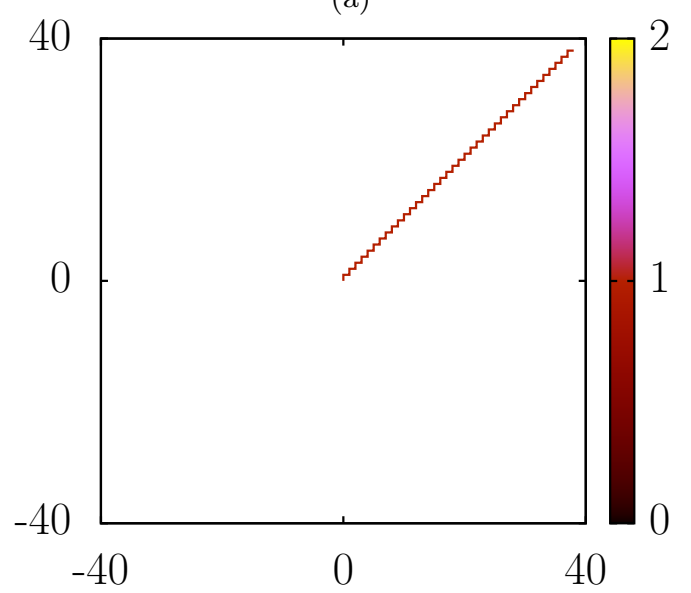

(b)

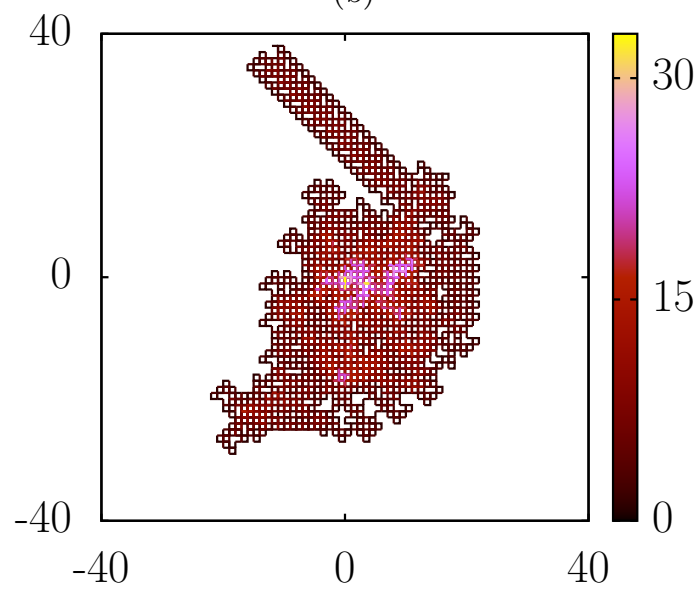

FIG. 1. (Color online). (a) A walk on an initially ordered flipping mirror environment of right mirrors in $\mathbb{Z}^{2}$. The walker alternates between a vertical and a horizontal step. (b) A walk on an initially ordered flipping rotator environment of right rotators in $\mathbb{Z}^{2}$, showing the visited sites. After 9,977 time steps the walker moves on a "highway" advancing two sites horizontally and two vertically every 104 time steps. In (a) and (b), at time $t=0$ the walker is at the origin with velocity $(1,0)$. (The color scale indicates the number of times the walker is at a site.)

region and walk on a highway, Fig. 2 (b).

The previous Figs. show that in $\mathbb{Z}^{2}$, walks on mirror environments are very different from from those on rotator environments. For walks in triangular lattices, $\mathbb{T}^{2}$, mirror and rotator scatterers can be defined but walks on the two environments are not so strikingly different. Again, mirror and rotator scatterers can be defined for walks in hexagonal lattices, $\mathbb{H}^{2}$, and walks on both environments are very different.

In this paper we are interested in the scattering rules of flipping mirror and flipping rotator environments in the three types of lattices, as defined in the literature. As we prove, the choice is justified by the fact that these are the only injective scattering rules meaning that walk- (a)

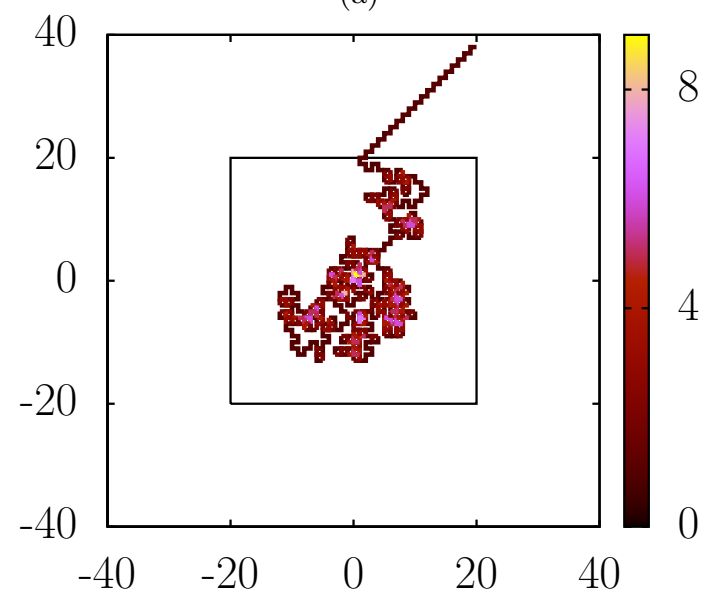

(b)

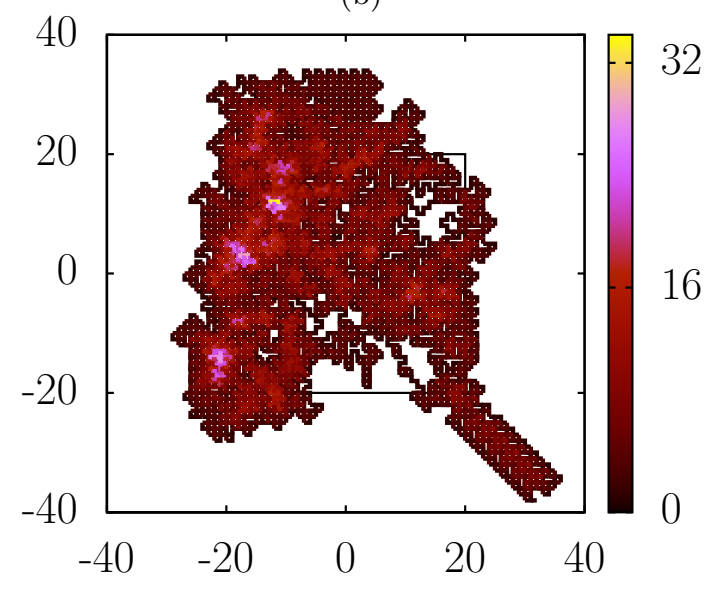

FIG. 2. (Color online). In (a) and (b), at time $t=0$, the environment is initially ordered with right scatterers outside the square of side 40 and inside this square the probability that any given site has a right scatterer is $1 / 2$. Initially, the walker is at the origin with velocity $(1,0)$. (a) A walk on a flipping mirror environment. (b) A walk on a flipping rotator environment. (The color scale indicates the number of times the walker is at a site.)

ers arriving to a scatterer with different velocities, will leave the site with different velocities. In the case of a flipping mirror environment on $\mathbb{Z}^{2}$ and $\mathbb{T}^{2}$ the walks are time reversible if in the time reversed walk the scatterer flips before scattering the walker, and in the other cases, walks are time reversible if in the time reversed walk the scatterer flips after the walker is scattered.

Given an initial position and velocity of a walker on one environment with one type of scatterers in $\mathbb{H}^{2}$, B. Z. Webb and E. G. D. Cohen proved that there is an environment with the other type of scatterers such that the walks on both environments are equivalent in the sense that their trajectories, $\{(x(t), y(t)) \mid t \in \mathbb{N}\}$ are the same [1]. Also, L. A. Bunimovich and S. E. Troubetzkoy stated the equivalence of walks on mirror and rotator environments with scatterers that do not flip in $\mathbb{Z}^{2}$, [12]. 
Our aim is to prove the equivalence of walks on square $\mathbb{Z}^{2}$ and triangular $\mathbb{T}^{2}$ lattices with flipping scatterers.

In Sec. II we present the walks on mirror and rotator environments in $\mathbb{Z}^{2}$, show that these are the only injective scattering rules, and prove the equivalence of walks as stated above. In the next Sec. we prove the same results for walks in $\mathbb{T}^{2}$ and for completeness, in Sec. IV, we also prove the equivalence of walks in $\mathbb{H}^{2}$. The proofs are based on an interplay between the geometry of the environment and the scattering rule. We close with some conclusions.

\section{WALKS IN $\mathbb{Z}^{2}$}

The main result of this Sec. is that given the initial position and velocity of a walker on an environment of scatterers, mirrors or rotators, in $\mathbb{Z}^{2}$, there is an environment with the other type of scatterers such that both walks are equivalent. We start with some definitions, then illustrate and prove the result.

The walker moves with one of four velocities $\boldsymbol{v}_{0}=$ $(1,0), \boldsymbol{v}_{1}=(0,1), \boldsymbol{v}_{2}=(-1,0)$, or $\boldsymbol{v}_{3}=(0,-1)$ in discrete time steps from one site on the environment to one of its nearest neighbor sites according to his/her velocity and the state of the scatterer he/she encounters. The environment is defined by $E=\left\{\sigma(x, y) \mid(x, y) \in \mathbb{Z}^{2}\right\}$ where $\sigma(x, y) \in\{-1,1\}$ is the state of the scatterer at $(x, y)$. When $\sigma(x, y)=1$ we say we have a right scatterer at $(x, y)$ and when $\sigma(x, y)=-1$ a left scatterer. Environments of mirrors and rotators will be denoted by $E_{M}$ and $E_{R}$ respectively in what follows. Right and left mirrors are shown schematically in Figs. 3 (a) and (c) and Figs. 4 (b) and (d). The reflection on the mirror forces the walker to turn an angle of $\pm \pi / 2$. A right (left) rotator scatters the walker an angle of $\pi / 2$ to his/her right (left) as we show in Figs. 3 (b) and (d) and Figs. 4 (a) and (c). After being scattered in either environment at $(x, y)$, the walker moves to one of two neighboring sites and $\sigma(x, y)$ flips by changing sign.

A walker with velocity $\boldsymbol{v}_{k}$ is scattered with velocity $\boldsymbol{v}_{k^{\prime}}$ with $k, k^{\prime}=0, \ldots, 3$. There are 4 ! different injective scattering rules since if $\boldsymbol{v}_{k} \neq \boldsymbol{v}_{l}$ then $\boldsymbol{v}_{k^{\prime}} \neq \boldsymbol{v}_{l^{\prime}}$. Of these, we chose those that scatter the walker an angle of $\pm \pi / 2$, that limits the scattering rules to four as we show in Table I. The four rules of Table I are equivalent in couples so effectively, there are only two injective scattering rules as we show next. The second and third columns of the Table show the scattering rule for mirrors $M$. A walker moving horizontally to the right, $k=0$, will be scattered vertically upwards when $\sigma=1, k^{\prime}=1$, and vertically downwards when $\sigma=-1, k^{\prime}=3$. A walker moving horizontally to the left, $k=2$, will be scattered vertically downwards, when $\sigma=1, k^{\prime}=3$, and vertically upwards when $\sigma=-1, k^{\prime}=1$. Thus, the first row of the scattering rule for a mirror fixes the values of the third row. This is also valid for the second and fourth rows of the scattering rule for $M$.
TABLE I. Scattering rules for mirrors, $M$, and rotators, $R$, in $\mathbb{Z}^{2}, \boldsymbol{v}_{k} \rightarrow \boldsymbol{v}_{k^{\prime}}$ with $k, k^{\prime}=0,1,2,3$. The other two possible scattering rules are $A$ and $B$, with $A_{-1}, A_{1}$, and $B_{-1}, B_{1}$ the orientations of the scatterers.

\begin{tabular}{|c|cc|cc|cc|cc|}
\hline$k$ & \multicolumn{9}{|c|}{$k^{\prime}$} \\
\hline & \multicolumn{2}{|c|}{$M$} & \multicolumn{2}{|c|}{$R$} & \multicolumn{2}{|c|}{$A$} & \multicolumn{2}{c|}{$B$} \\
\hline & $\sigma=1$ & $\sigma=-1$ & $\sigma=1$ & $\sigma=-1$ & $A_{-1}$ & $A_{1}$ & $B_{-1}$ & $B_{1}$ \\
\hline 0 & 1 & 3 & 3 & 1 & 3 & 1 & 1 & 3 \\
1 & 0 & 2 & 0 & 2 & 2 & 0 & 2 & 0 \\
2 & 3 & 1 & 1 & 3 & 1 & 3 & 3 & 1 \\
3 & 2 & 0 & 2 & 0 & 0 & 2 & 0 & 2 \\
\hline
\end{tabular}

The fourth and fifth columns of Table I show the scattering rule for rotators $R$. A right rotator, $\sigma=1$, scatters the walker to his/her right and a left rotator, $\sigma=-1$, to his/her left. Again, the first and second rows fix the third and fourth ones respectively. The other two scattering rules, called $A$ and $B$ are shown in the remaining columns of Table I where $A_{-1}$ and $A_{1}$ are the two states of the $A$ scatterer and $B_{-1}$ and $B_{1}$ those of the $B$ scatterer. With $A_{-1}=-1$ and $A_{1}=1$, the $A$ scattering rule is the same as that of the mirror $M$, and with $B_{-1}=-1$, $B_{1}=1$, the $B$ scattering rule is that of the rotator $R$. Hence, there are only two injective rules that scatter the walker by angles of $\pm \pi / 2$.

An initially ordered environment is one with $\sigma(x, y)=$ $1 \forall(x, y) \in \mathbb{Z}^{2}$, or equivalently $\sigma(x, y)=-1 \forall(x, y) \in$ $\mathbb{Z}^{2}$. In what follows and without loss of generality we chose the first option. A checkerboard environment is one in which at any site $(x, y)$, the sign of the scatterers of the four nearest neighbor sites is opposite to that at $(x, y)$. Given the initial position of the walker at the origin, there are two checkerboard environments, one with $\sigma(0,0)=1$, the other one with $\sigma(0,0)=-1$.

The first example of this Sec. is that a walk initially at $(0,0)$ with velocity $(1,0)$ on an initially ordered flipping mirror environment, $O F M E$, is equivalent to a walk that starts with the same initial position and velocity on an initially checkerboard flipping rotator environment, $C F R E$, provided this environment is chosen in such a way that at time $t=0$ both walkers are scattered in the same direction. The second example is the equivalence of trajectories on an initially ordered flipping rotator environment, $O F R E$, and on an initially checkerboard flipping mirror environment, CFME, when the initial position and velocity of both walkers satisfy the imposed conditions of the first example.

In Figs. 3 (a) and (b) we show a OFME and a $C F R E$ respectively. The initial position of the walkers is at $(0,0)$ marked by a small open circle. In Fig. 3 (c) we show the walk and the state of the mirror environment at time $t=6$, and in Fig. 3 (d) the walk and the state of the the rotator environment at the same time. Both walkers at $t=0$ have $\boldsymbol{v}_{0}=(1,0)$ and are scattered upwards as shown by the arrow from $(0,0)$ to $(0,1)$. At $t=1$, both 
(a)

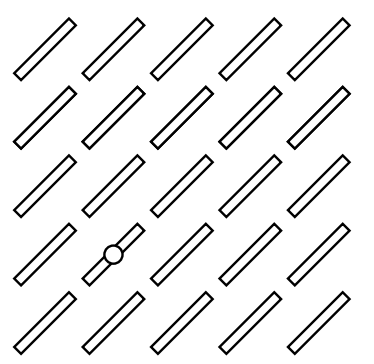

(c)

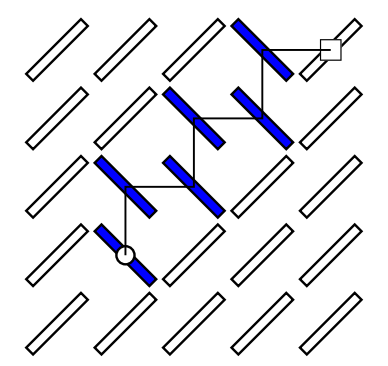

(b)

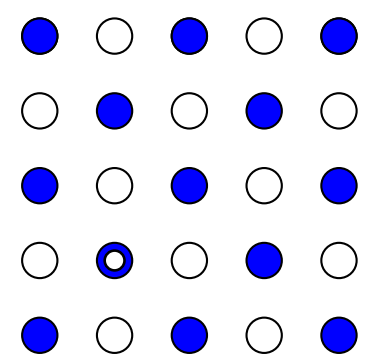

(d)

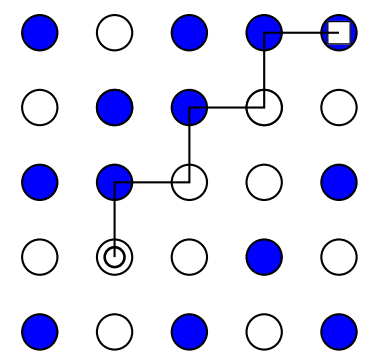

FIG. 3. (Color online). (a) Initially ordered flipping mirror environment, $O F M E$. (b) Initially checkerboard flipping rotator environment, CFRE. (c) A walk on the $O F M E$ at $t=6$ showing the walk and the state of the environment. (d) A walk on the CFRE at $t=6$ showing the walk and the state of the environment. In both cases, at $t=0$ the walker is at the site marked by a small open circle and has velocity $\boldsymbol{v}_{0}=(1,0)$ and at $t=6$, the walker's position is marked by a small open square. Right mirrors make an angle of $\pi / 4$ with the positive $x$ axis, shown as open rectangles (in white). Left mirrors make an angle of $3 \pi / 4$ with the positive $x$ axis, shown as dark rectangles (in blue). Right and left rotators are shown as open circles (in white) and dark circles (in blue) respectively.

walkers are at $(0,1)$ and are scattered to their right and at $t=2$ arrive at $(1,1)$. Both walkers are then scattered upwards and then horizontally to the right reaching $(3,3)$ at $t=6$, shown by an open square. Although the scatterers flip after the walker passes, they do not influence the walk. Thus, both walkers follow the same trajectory alternating between a vertical and a horizontal step and moving diagonally with a speed of $\sqrt{2} / 2$.

In Figs. 4 (a) and (b) we show a OFRE and a $C F M E$ respectively. The initial position of the walkers on both environments is shown by a small open circle at $(0,0)$. In Fig. 4 (c) we show the walk and the state of the rotator environment at $t=6$ and in Fig. 4 (d) the walk and the state of the mirror environment at the same time. Both walkers are at $(0,0)$ with velocity $\boldsymbol{v}_{0}=(1,0)$ at $t=0$. In the rotator environment, Fig. 4 (c), the walker is first scattered to his/her right and moves downward reaching $(0,-1)$ at $t=1$, is again scattered to his/her right to $(-1,-1)$ at $t=2$, again to his/her right to $(-1,0)$ at $t=3$, and at $t=4$ is back at the origin with velocity $\boldsymbol{v}_{0}$. The same happens in the mirror environment, Fig. 4 (d),

(a)

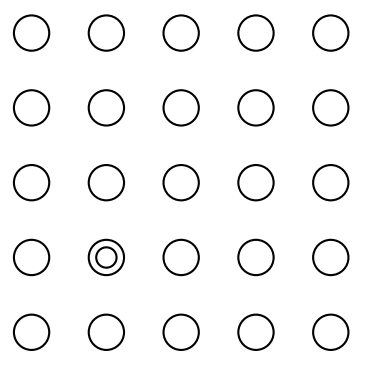

(c)

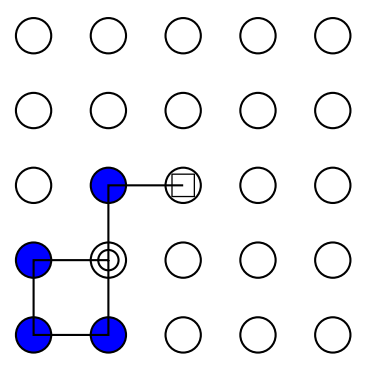

(b)

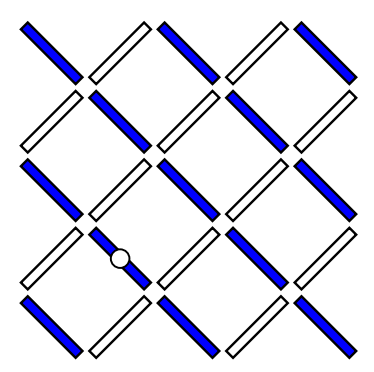

(d)

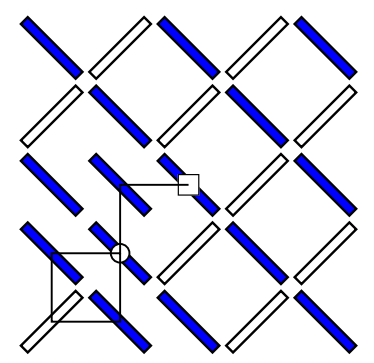

FIG. 4. (Color online). (a) Initially ordered flipping rotator environment, $O F R E$. (b) Initially checkerboard flipping mirror environment, $C F M E$. (c) A walk on the $O F R E$ at $t=6$. (d) A walk on the $C F M E$ at $t=6$. The convention on the initial and final positions and initial velocity of both walkers and the scatterers is the same as in the previous Fig.

the walker is scattered to his/her right from $t=0$ to $t=4$ when he/she is back at the origin. Since both scatterers at $(0,0)$ have flipped, both walkers will be scattered to their left and move upwards and then they will scatter to their right so that at $t=6$ are at $(1,1)$. Both walkers will scatter to their right at sites that are visited for the first time, to their left at sites that have been visited once, again to their right at sites that have been visited two times and so on. Thus, their trajectories are equivalent up to time $t=6$. In Fig. 1 (b) we show the walk on a $O F R E$ for a longer time.

In Theorem 1 we show that given a walk on one environment, there is an equivalent walk on the other one. Two walks are equivalent if their paths $\{(x(t), y(t)) \mid t \in$ $\mathbb{N}\}$ are the same.

Theorem 1 Let $E_{M}=\left\{\sigma_{M}(x, y) \mid(x, y) \in \mathbb{Z}^{2}\right\}$ be a mirror environment, $E_{R}=\left\{\sigma_{R}(x, y) \mid(x, y) \in \mathbb{Z}^{2}\right\}$ a rotator environment, and $d(x, y)=|x|+|y|$ for any $(x, y) \in \mathbb{Z}^{2}$. Given a walk with initial position $(0,0)$ and velocity $\boldsymbol{v}_{0}$ on one environment, there is an equivalent walk with the same initial position and velocity on the other environment if

$$
\sigma_{R}(x, y)=(-1)^{d(x, y)+1} \sigma_{M}(x, y) .
$$

Proof of Theorem 1. A site $(x, y)$ is even (odd) if $d$ is even (odd). The number of steps of any path from the origin to an even (odd) site is even (odd). Since the initial 
velocity $\boldsymbol{v}_{0}$ is horizontal, the velocity of the walker at odd times will be vertical and at even times horizontal, that is $\boldsymbol{v}(2 t+1)$ is either $\boldsymbol{v}_{1}$ or $\boldsymbol{v}_{3}$ and $\boldsymbol{v}(2 t)$ is either $\boldsymbol{v}_{0}$ or $\boldsymbol{v}_{2}$ for $t=0,1, \ldots$ This implies that the walker arrives to even sites with a horizontal velocity, $\boldsymbol{v}_{0}$ or $\boldsymbol{v}_{2}$, and to odd sites with a vertical velocity, $\boldsymbol{v}_{1}$ or $\boldsymbol{v}_{3}$.

From Table I we get that if the velocity is $\boldsymbol{v}_{1}$ or $\boldsymbol{v}_{3}$ the mirror and rotator scatterers of the same sign act in the same way on the walker, but if the velocity is $\boldsymbol{v}_{0}$ or $\boldsymbol{v}_{2}$ the mirror and rotator scatterers of the same sign act in opposite ways on the walker. Thus to change an environment of rotators for one of mirrors, or the inverse, in such a way that the walkers follow the same trajectory, we have to change the sign of the scatterers at even sites. This implies the result.

If one of the environments is initially ordered, the other one is a checkerboard according to the theorem. We finish with a remark on the initial conditions of the walker. If the starting point is $\left(x_{0}, y_{0}\right)$ the function $d(x, y)$ has to be replaced by the function $d_{\left(x_{0}, y_{0}\right)}(x, y)=$ $\left|\left(x-x_{0}\right)\right|+\left|\left(y-y_{0}\right)\right|$. This change implies that the exponent of -1 in Eq. (1) is replaced by $d_{\left(x_{0}, y_{0}\right)}(x, y)+1$. If the initial velocity is changed to $\boldsymbol{v}_{2}$, the rule for changing the environment is the same as in Theorem 1, but if it is $\boldsymbol{v}_{1}$ or $\boldsymbol{v}_{3}$ the exponent of -1 has to be replaced by $d_{\left(x_{0}, y_{0}\right)}(x, y)$ as can be deduced from the proof above.

\section{WALKS IN $\mathbb{T}^{2}$}

We consider walks on flipping environments in $\mathbb{T}^{2}$ where the walker is scattered by angles of $\pm 2 \pi / 3$ with respect to his/her velocity and show that there are only two injective scattering rules. We then prove that given the initial position and velocity of a walker in an environment with one type of scatterers, there is an environment with the other type of scatterers such that the walks on the two environments are equivalent.

The six possible velocities in $\mathbb{T}^{2}$, with $h=\sqrt{3} / 2$, are $\boldsymbol{v}_{0}=(1,0), \boldsymbol{v}_{1}=(1 / 2, h), \boldsymbol{v}_{2}=(-1 / 2, h), \boldsymbol{v}_{3}=(-1,0)$, $\boldsymbol{v}_{4}=(-1 / 2,-h$,$) , and \boldsymbol{v}_{5}=(1 / 2,-h)$. In one time step, a walker with velocity $\boldsymbol{v}_{k}$ is scattered by angles of $\pm 2 \pi / 3$ with velocity $\boldsymbol{v}_{k^{\prime}}$ with respect to $\boldsymbol{v}_{k}$ and moves to one of two possible neighbor sites according to one of the injective rules shown in Table II. If $k$ is odd (even), $k^{\prime}$ is odd (even). The scattering rules for mirrors and rotators are shown in the columns $M$ and $R$ of Table II, respectively, corresponding to models $2 \mathrm{~B}$ and $1 \mathrm{~B}$ of Ref.[17]. In the Table, the columns $A$ and $B$ are the other two possible injective scattering rules. For the $A$ rule, with $A_{-1}=-1$ and $A_{1}=1$ we obtain the mirror $M$ scattering rule, and for the $B$ rule, $B_{-1}=-1$ and $B_{1}=1$ gives the rotator $R$ scattering rule. Thus there are only two injective scattering rules on $\mathbb{T}^{2}$ for which the walker turns by angles of $\pm 2 \pi / 3$. In Fig. 5 we show walks on initially ordered flipping mirror and flipping rotator environments. In both cases, after a small transient, the walker advances half a site horizontally and one site vertically every 8 time
TABLE II. Scattering rules for mirrors, $M$, and rotators, $R$, in $\mathbb{T}^{2}, \boldsymbol{v}_{k} \rightarrow \boldsymbol{v}_{k^{\prime}}$ with $k, k^{\prime}=0, \ldots, 5$. The other two possible scattering rules are $A$ and $B$, with $A_{-1}, A_{1}$, and $B_{-1}, B_{1}$ the orientations of the scatterers.

\begin{tabular}{|c|cc|cc|cc|cc|}
\hline$k$ & \multicolumn{9}{|c|}{$k^{\prime}$} \\
\hline & \multicolumn{2}{|c|}{$M$} & \multicolumn{2}{|c|}{$R$} & \multicolumn{2}{|c|}{$A$} & \multicolumn{2}{c|}{$B$} \\
\hline & $\sigma=1$ & $\sigma=-1$ & $\sigma=1$ & $\sigma=-1$ & $A_{-1}$ & $A_{1}$ & $B_{-1}$ & $B_{1}$ \\
\hline 0 & 2 & 4 & 4 & 2 & 4 & 2 & 2 & 4 \\
1 & 5 & 3 & 5 & 3 & 3 & 5 & 3 & 5 \\
2 & 4 & 0 & 0 & 4 & 0 & 4 & 4 & 0 \\
3 & 1 & 5 & 1 & 5 & 5 & 1 & 5 & 1 \\
4 & 0 & 2 & 2 & 0 & 2 & 0 & 0 & 2 \\
5 & 3 & 1 & 3 & 1 & 1 & 3 & 1 & 3 \\
\hline
\end{tabular}
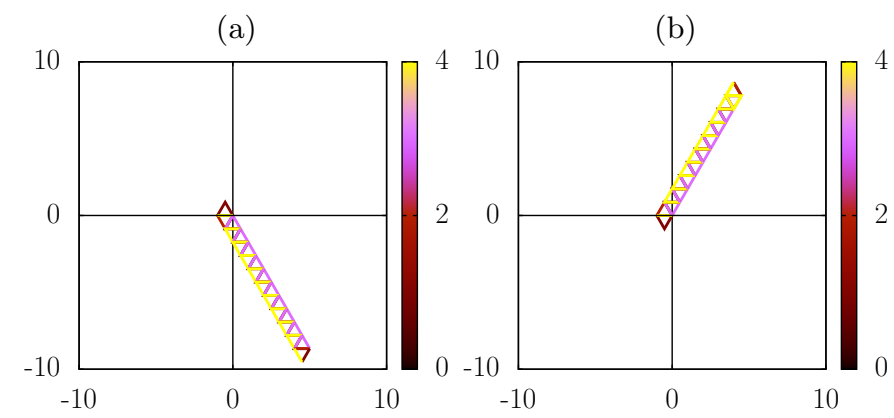

FIG. 5. (Color online). Walks on initially ordered environments with with $\sigma(x, y)=1$ on all sites. (a) Flipping mirror environment $O F M E$ and (b) flipping rotator environment $O F R E$. In both cases, at $t=0$, the walker is at $(0,0)$ with velocity $\boldsymbol{v}_{0}$. (The color scale indicates the number of times the walker is at a site.)

steps, moving with a speed of $\sqrt{5} / 16$ in the direction of $\boldsymbol{v}_{5}$ in the mirror environment and in the direction of $\boldsymbol{v}_{1}$ in the rotator environment. On initially disordered environments, the walker will also move in strips as shown by Grosfils et al [18]. The walks on mirror and rotator environments are not so strikingly different as those in $\mathbb{Z}^{2}$. In Theorem 2 we prove the equivalence of walks on mirror and rotator environments.

Theorem 2 Let $E_{M}=\left\{\sigma_{M}(x, y) \mid(x, y) \in \mathbb{T}^{2}\right\}$ be a mirror environment and $E_{R}=\left\{\sigma_{R}(x, y) \mid(x, y) \in \mathbb{T}^{2}\right\}$ a rotator environment.

(a) For every walk on one environment with some initial position and initial velocity $\boldsymbol{v}_{0}=(1,0)$, $\boldsymbol{v}_{2}=(-1 / 2, h)$ or $\boldsymbol{v}_{4}=(-1 / 2,-h$,$) there is an$ equivalent walk on the other environment with the same initial position and velocity of the walker, if $\sigma_{R}(x, y)=-\sigma_{M}(x, y) \forall(x, y) \in \mathbb{T}^{2}$.

(b) For every walk on one environment with some initial position and initial velocity $\boldsymbol{v}_{1}=(1 / 2, h)$, $\boldsymbol{v}_{3}=(-1,0)$, or $\boldsymbol{v}_{5}=(1 / 2,-h)$ there is an equivalent walk on the other environment with the 
same initial position and velocity of the walker, if $\sigma_{R}(x, y)=\sigma_{M}(x, y) \forall(x, y) \in \mathbb{T}^{2}$.

Proof of Theorem 2.

(a) Given that the walker has an initial velocity $\boldsymbol{v}_{k}$ with $k$ even, the velocity at any time step will also have $k$ even on both environments as shown in Table II. The columns in the rows of Table II corresponding to $k$ even and the mirror scattering rule are inverted with respect to those of the rotator rule. Then walks on the mirror and the rotator environments with the same initial position and velocity $\boldsymbol{v}_{k}$ are equivalent if $\sigma_{R}(x, y)=-\sigma_{M}(x, y)$.

(b) Given that the walker has an initial velocity $\boldsymbol{v}_{k}$ with $k$ odd, the velocity at any time step will also have $k$ odd on both environments as shown in Table II. The mirror and scattering rules in Table II coincide for $k$ odd. Then walks on the two environments with the same initial position and velocity $\boldsymbol{v}_{k}$, for $k$ odd, are equivalent if $\sigma_{R}(x, y)=\sigma_{M}(x, y)$.

\section{WALKS IN $\mathbb{H}^{2}$}

As mentioned in Sec. I, the equivalence of walks in $\mathbb{H}^{2}$ was proven in Ref, [1]. For completeness, we present a proof of the same result. A walker has a velocity $\boldsymbol{v}_{k}$, $k=0, \ldots, 5$ as in the previous Sec. and is scattered at every time step by angles of $\pm \pi / 3$. Depending on the value of the initial velocity, the walker will visit a different hexagonal two dimensional lattice $\mathbb{H}^{2}$. We show, as in the previous Secs., that there are only two injective scattering rules, one gives way to a rotator environment and the other one to a mirror environment. We show that walks on mirror and rotator environments in $\mathbb{H}^{2}$ are qualitatively different and prove that for a walk with fixed initial position and velocity on one environment, there is an equivalent walk with the same initial conditions on the other environment.

In Table III we show the four injective scattering rules. A walker with velocity $\boldsymbol{v}_{k}$ is scattered with velocity $\boldsymbol{v}_{k^{\prime}}$. If $k$ is even (odd), $k^{\prime}$ is odd (even) for the four rules. In the mirror rule, shown in the $M$ columns of the Table, if $\boldsymbol{v}_{k}$ is scattered to $\boldsymbol{v}_{k^{\prime}}$, then $\boldsymbol{v}_{k^{\prime}}$ is scattered to $\boldsymbol{v}_{k}$ for the same value of $\sigma$. This also happens for walks in mirror environments in $\mathbb{Z}^{2}$. The rotator rule is shown in the $R$ columns of the Table, a walker with velocity $\boldsymbol{v}_{k}$ is scattered with velocity $\boldsymbol{v}_{k^{\prime}}$ with $k^{\prime}=(k-\sigma) \bmod 6$. As in the previous cases the rules are equivalent by couples. If $A_{-1}=-1, A_{1}=1$, we obtain the mirror $M$ scattering rule, and if $B_{-1}=-1, B_{1}=1$, we obtain the rotator $R$ scattering rule.

In Fig. 6 (a) we show a walk on an initially ordered flipping mirror environment $O F M E$ in $\mathbb{H}^{2}$. The walker moves alternatively in directions $\boldsymbol{v}_{5}$ and $\boldsymbol{v}_{0}$ with a speed of $\sqrt{3} / 2$. When there is some disorder in the initial environment of mirrors, the walker moves as we show in
TABLE III. Scattering rules for mirrors, $M$, and rotators, $R$, in $\mathbb{H}^{2}, \boldsymbol{v}_{k} \rightarrow \boldsymbol{v}_{k^{\prime}}$ with $k, k^{\prime}=0, \ldots, 5$. The other two possible scattering rules are $A$ and $B$, with $A_{-1}, A_{1}$, and $B_{-1}, B_{1}$ the orientations of the scatterers.

\begin{tabular}{|c|cc|cc|cc|cc|}
\hline$k$ & \multicolumn{9}{|c|}{$k^{\prime}$} \\
\hline & \multicolumn{2}{|c|}{$M$} & \multicolumn{2}{|c|}{$R$} & \multicolumn{2}{|c|}{$A$} & \multicolumn{2}{c|}{$B$} \\
\hline & $\sigma=1$ & $\sigma=-1$ & $\sigma=1$ & $\sigma=-1$ & $A_{-1}$ & $A_{1}$ & $B_{-1}$ & $B_{1}$ \\
\hline 0 & 5 & 1 & 5 & 1 & 1 & 5 & 1 & 5 \\
1 & 2 & 0 & 0 & 2 & 0 & 2 & 2 & 0 \\
2 & 1 & 3 & 1 & 3 & 3 & 1 & 3 & 1 \\
3 & 4 & 2 & 2 & 4 & 2 & 4 & 4 & 2 \\
4 & 3 & 5 & 3 & 5 & 5 & 3 & 5 & 3 \\
5 & 0 & 4 & 4 & 0 & 4 & 0 & 0 & 4 \\
\hline
\end{tabular}

(a)

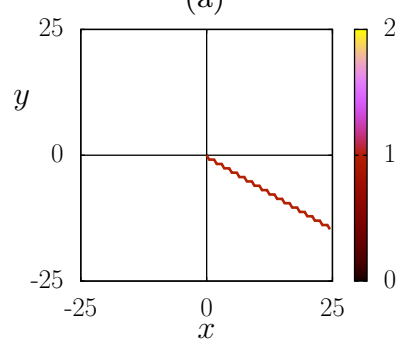

(c)

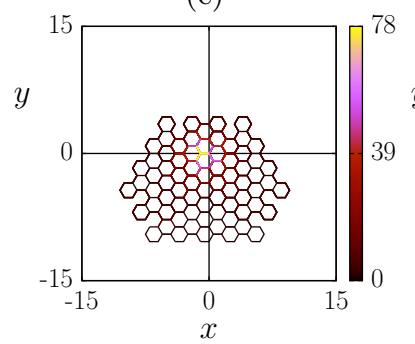

(b)

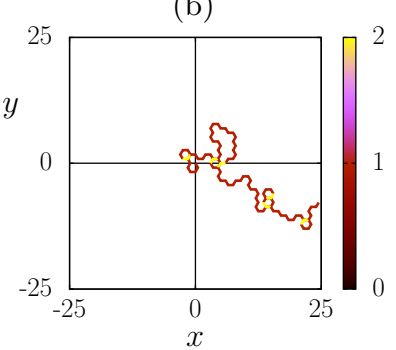

(d)

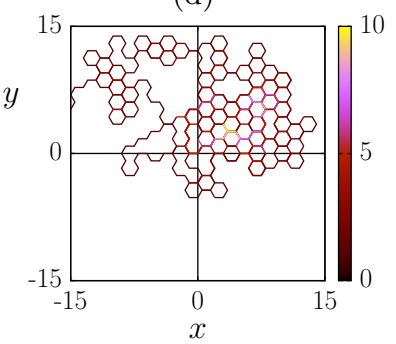

FIG. 6. (Color online.) (a) and (b) Trajectories of a walker on flipping mirror environments in $\mathbb{H}^{2}$. In (a), the environment is initially ordered with right mirrors. In (b), at $t=0$, the environment is ordered outside the square of side 40 and inside this square the probability that any given site has a right mirror is $1 / 2$. (c) and (d) Trajectories of a walker on flipping rotator environments in $\mathbb{H}^{2}$. In (c), the environment is initially ordered with right rotators. In (d), at $t=0$, the environment is ordered with right rotators outside the square of side 40 , and inside this square the probability that any given site has a right rotator is $1 / 2$. In the four cases, the walker is initially at $(0,0)$ with velocity $\boldsymbol{v}_{0}=(1,0)$. (The color scale indicates the number of times the walker is at a site.)

Fig. 6 (b). A walk on an initially ordered flipping rotator environment $O F R E$ is completely different as we show in Fig. 6 (c), the walk is self-avoiding between returns to the origin [1]. In Fig. 6 (d) we show a walk on an initially disordered flipping rotator environment.

For a walk on one of the environments in $\mathbb{H}^{2}$, we prove in Theorem 3 that there is an equivalent walk on the other environment. 
Theorem 3 Let $E_{M}=\left\{\sigma_{M}(x, y) \mid(x, y) \in \mathbb{H}^{2}\right\}$ be a mirror environment, $E_{R}=\left\{\sigma_{M}(x, y) \mid(x, y) \in \mathbb{H}^{2}\right\}$ a rotator environment, and $d(x, y)$ the number of steps of the shortest path (which is not in general unique) from $(0,0)$ to $(x, y)$.

(a) If

$$
\sigma_{M}(x, y)=(-1)^{d(x, y)} \sigma_{R}(x, y),
$$

walks that begin at $(0,0)$ with velocity $\boldsymbol{v}_{0}=(1,0)$, $\boldsymbol{v}_{2}=(-1 / 2, h)$ or $\boldsymbol{v}_{4}=(-1 / 2,-h)$ on both environments are equivalent. That is, $\{(x(t), y(t)) \mid t \in$ $\mathbb{N}\}$ is the same for both walks.

(b) If

$$
\sigma_{M}(x, y)=(-1)^{d(x, y)+1} \sigma_{R}(x, y),
$$

walks that begin at $(0,0)$ with velocity $\boldsymbol{v}_{1}=$ $(1 / 2, h), \boldsymbol{v}_{3}=(-1,0)$ or $\boldsymbol{v}_{5}=(1 / 2,-h)$ on both environments are equivalent. That is, $\{(x(t), y(t)) \mid t \in$ $\mathbb{N}\}$ is the same for both walks.

Proof of Theorem 3. If $d$ is an even (odd) number, $(x, y)$ is an even (odd) site of $\mathbb{H}^{2}$. The nearest neighbors of an even (odd) site are odd (even) sites. Note that if $(x, y)$ is an even (odd) site, any walk from the origin to $(x, y)$ will visit an even (odd) number of sites. From Table III, a walk with initial velocity $\boldsymbol{v}_{k}$ and $k$ even will have a velocity with $k$ odd at odd times and a velocity with $k$ even at even times. Also from Table III we have that $M$ and $R$ scatterers with the same sign of $\sigma$ scatter the walker in the same direction $k^{\prime}$ if $k$ is even and opposite directions if $k$ is odd.

Combining these two observations we obtain that if the initial velocity has $k$ even, $\boldsymbol{v}_{0}, \boldsymbol{v}_{2}$ or $\boldsymbol{v}_{4}$, the scatterers at odd sites $(x, y)$ must have opposite signs in the two environments in order to scatter the walker in the same direction. This proves part (a) of the Theorem. Analogously, if the initial velocity has odd $k, \boldsymbol{v}_{1}, \boldsymbol{v}_{3}$ or $\boldsymbol{v}_{5}$, scatterers at even sites have to have opposite signs in the $M$ and $R$ environments, proving part (b) of the Theorem.

\section{CONCLUDING REMARKS}

We showed that on the three regular lattices on the plane, square, triangular, and hexagonal, there are only two injective two state scattering rules, rotators and mirrors. We extended Webb and Cohen's result of the equivalence of walks on flipping mirror and rotator environments in hexagonal lattices to the equivalence of walks on triangular and square lattices. The proofs of the equivalence of walks on both environments are based on an interplay between the scattering rules and the geometry of the lattice.

Given a walk on an initially ordered flipping rotator environment OFRE in $\mathbb{H}_{2}$, Cohen and Webb [1] proved that the walk is self-avoiding between successive returns to the origin. As a consequence of the equivalence of walks, a walk on an initially checkerboard flipping mirror environment $C F M E$ in $\mathbb{H}_{2}$ will be self-avoiding between successive returns to the origin. In [14], Webb and Cohen study the trajectories on flipping rotator environments. Starting with an initially ordered flipping rotator environment (of right or left rotators), they prove that for a walk in an initial environment obtained by changing the rotator at each site with probability $p \in(0,1)$, then the trajectory starting at $(0,0)$ with velocity $\boldsymbol{v}_{0}$ will be periodic with probability 1 . Applying Theorem 3 we can state this result for flipping mirror environments: starting with an initially checkerboard flipping mirror environment, consider a walk in an initial environment obtained by changing the mirror at each site with probability $p \in(0,1)$, then the trajectory starting at $(0,0)$ with velocity $\boldsymbol{v}_{0}$ will be periodic with probability 1 .

For any walk on any environment with one type of scatterers in one of the three two dimensional regular lattices, there is an equivalent walk on another environment with the other type of scatterers. This means, that whatever result is valid for walks with one type of scatterers is valid for walks with the other type of scatterers if the second environment is chosen according to the theorems proved above.

\section{ACKNOWLEDGMENTS}

It is a pleasure to acknowledge many enlightening discussions with E. G. D. Cohen, H. Larralde, and M. López de Haro. Also, interesting discussions with H. D. Cortés González and M. Valdez González are acknowledged. We also acknowledge the anonymous reviewers. Their comments have enriched this presentation. A. Rechtman acknowledges the support of LAISLA, the collaboration program between Mexico and France for mathematics.
[1] B. Z. Webb and E. G. D Cohen. Self-avoiding modes of motion in a deterministic Lorentz lattice gas. J. Phys. A: Math. Theor, 47:315202, 2014.

[2] P. Ehrenfest and T. Ehrenfest. Begriffliche Grundlagen der statistische Auffasung in der Mechanik. In Encyklopädie der Mathematische Wissenschaften, volume
IV:2:II, No. 6. B. G. Teubner, (Leipzig), 1912. Translated to English by M. J. Moravcsik in The Conceptual Foundations of the Statistical Approach in Mechanics, Cornell University Press, Ithaca NY, (1959).

[3] L. A. Bunimovich. Deterministic walks in random environments. Physica D, 187:20, 2004. 
[4] Th. W. Ruijgrok and E. G. D. Cohen. Deterministic lattice gas models. Phys. Lett. A, 133:415, 1988.

[5] E. G. D. Cohen. New types of diffusion in lattice gas cellular automata. In M.Mareschal and B. Holian, editors, Microscopic Simulations of Complex Hydrodynamic Phenomena, volume NATO ASI Series B, vol 292, 1992.

[6] J. M. F. Gunn and M. Ortuno. Percolation and motion in a simple random environment. J. Phys. A, 18:L1095, 1985.

[7] C. G. Langton. Studying artificial life with cellular automata. Physica D, 22:120, 1986.

[8] Hsin-Fei Meng and E. G. D. Cohen. Growth, selfrandomization, and propagation in a Lorentz lattice gas. Phys. Rev. E, 50:2482, 1994.

[9] X. P. Kong and E. G. D. Cohen. Lorentz lattice gases, abnormal diffusion, and polymer statistics. J. Stat. Phys., 62:1153, 1991.

[10] E. G. D. Cohen and F.Wang. New results for diffusion in Lorentz lattice gas cellular automata. J. Stat. Phys., 81:445, 1995 .

[11] E. G. D. Cohen and F.Wang. Novel phenomena in Lorentz lattice gases. Physica A, 219:56, 1995.
[12] L. A. Bunimovich and S. E. Troubetzkoy. Recurrence properties of Lorentz lattice gas cellular automata. $J$. Stat. Phys., 67:289, 1992.

[13] F. Wang and E. G. D. Cohen. Diffusion in Lorentz lattice gas cellular automata: the honeycomb and quasi-lattices compared with the square and triangular lattices. J. Stat. Phys, 81:467, 1995.

[14] B. Z. Webb and E. G. D Cohen. Self-limiting trajectories of a particle moving deterministically in a random medium. J. Phys. A: Math. Theor., 48:485203, 2014.

[15] D. Gale, J. Propp, and S. Troubetzkoy. Further travels with my ant. Math. Intelligencer, 17:48, 1995.

[16] J. P. Boon. How fast does Langton's ant move? J. Stat. Phys., 102:355, 2001.

[17] X. P. Kong and E. G. D. Cohen. Diffusion and propagation in triangular Lorentz lattice gas cellular automata. J. Stat. Phys., 62:737, 1991.

[18] P. Grosfils, J. P. Boon, E. G. D. Cohen, and L. A. Bunimovich. Propagation and organization in lattice random media. J. Stat. Phys., 97:575, 1999. 